\title{
Sistema de control automático de variables climáticas para optimizar el rendimiento de cultivos bajo cubierta
}

\author{
Automatic Control System for Climate Variables \\ to Optimize Greenhouse Crop Yields
}

\author{
Álvaro Hernán Alarcón-López ${ }^{1} \bowtie$, Geyni Arias-Vargas², \\ Cristian Javier Díaz-Ortiz ${ }^{3}$, Juan David Sotto-Vergara ${ }^{4}$ \\ ${ }^{1}$ Corporación Universitaria del Huila-Corhuila, Neiva, Colombia \\ ${ }^{2}$ Corporación Universitaria del Huila-Corhuila, Neiva, Colombia \\ ${ }^{3}$ Corporación Universitaria del Huila-Corhuila, Neiva, Colombia \\ ${ }^{4}$ Corporación Universitaria del Huila-Corhuila, Neiva, Colombia
}

Grupo de Investigación Innovación, investigación y desarrollo de proyectos de tecnologías de la información-Inproti, Facultad de Ingeniería, Corporación Universitaria del Huila-Corhuila. Calle 8 N. ${ }^{\circ}$ 32-49 (Prado Alto), Neiva, Huila. Correo electrónico: alvaro.alarcon@corhuila.edu.co

Recibido: 1 de septiembre del 2017 Aprobado: 1 de diciembre del 2017 Disponible en línea: 1 de enero del 2018

How to cite this article: A. H. Alarcón-López, G. Arias-Vargas, C. J. Díaz-Ortiz y J. D. Sotto-Vergara, "Sistema de control automático de variables climáticas para optimizar el rendimiento de cultivos bajo cubierta", Revista Ingeniería Solidaria, vol. 14, no. 24, pp. 11, enero 2018. doi: https://doi.org/10.16925/in.v14i24.2158

\section{Resumen}

Introducción: el artículo es resultado de la investigación "Diseño de un sistema de control y automatización de temperatura, humedad del suelo y humedad relativa para optimizar el rendimiento de cultivos bajo cubierta en Corhuila", desarrollada en la Corporación Universitaria del Huila entre el 2016 y el 2017.

Objetivo: mejorar los índices de crecimiento de las plantaciones al interior de un invernadero.

Metodología: el estudio se fundamentó en una comparación realizada a nivel de crecimiento y cantidad de frutos entre un cultivo de tomate ubicado bajo cubierta y uno situado a la intemperie; se realizó un seguimiento semanal de los ítems anteriormente mencionados mediante observación directa de los dos grupos de plantaciones.

Resultados: durante las primeras cinco semanas, se presentó un desarrollo similar; a partir de la séptima semana, el cultivo bajo cubierta presentó un $38 \%$ de mayor cantidad de ramas, igualdad a nivel de frutos y un 28\% de mayor altura; en la semana 13 las diferencias se elevaron al 64\% en cantidad de ramas, 65\% de mayor cantidad de frutos y $55 \%$ en altura.

Conclusión: se comprobó que la implantación de esta solución tecnológica puede propiciar un incremento del índice de crecimiento y producción alcanzado por las plantas.

Originalidad: desarrollo de un sistema electrónico para el sector agricultor de una región intermedia de Colombia, con el propósito de mejorar la eficiencia de los sembradíos.

Limitaciones: la carencia de acceso a Internet imposibilitó la implementación de un sistema control vía web.

Palabras clave: actuadores, automatización, cultivos, invernadero, sensores, software. 


\title{
Automatic Control System for Climate Variables to Optimize Greenhouse Crop Yields
}

\begin{abstract}
Introduction: The article derives from the research "Design of a system for the control and automation of temperature, soil moisture, and relative humidity to optimize greenhouse crop yields at Corhuila" conducted at Corporación Universitaria del Huila between 2016 and 2017.

Aim: To improve growth rates of greenhouse crops.

Methods: The study was based on a comparison of growth and number of fruits between greenhouse and outdoor tomato crops; said items were monitored weekly by direct observation of the two groups of crops.

Results: Development during the first five weeks was similar; as of the seventh week, the greenhouse crop had $38 \%$ more branches, equal number of fruits and was $28 \%$ higher; on week 13 , the differences increased to $64 \%$ in the number of branches, $65 \%$ in the number of fruits and $55 \%$ in height.

Conclusion: It was proved that the implementation of this technological solution can promote an increase in the growth and production rate reached by plants.

Originality: Development of an electronic system for the agricultural sector in an intermediate region of Colombia to improve the efficiency of crops.

Limitations: The lack of Internet access made it impossible to implement a web-based control system.
\end{abstract}

Keywords: actuators, automation, crops, greenhouse, sensors, software.

\section{Sistema de controle automático de variáveis climáticas para otimizar o rendimento de cultivos sob abrigo}

\section{Resumo}

Introdução: este artigo é resultado da pesquisa "Desenho de um sistema de controle e automatização de temperatura, umidade do solo e umidade relativa para otimizar o rendimento de cultivos sob abrigo em Corhuila", desenvolvida na Corporación Universitaria del Huila entre 2016 e 2017.

Objetivo: melhorar os índices de crescimento das plantações no interior de uma estufa.

Metodologia: o estudo foi fundamentado numa comparação realizada quanto a crescimento e quantidade de frutos entre um cultivo de tomate localizado sob abrigo e um situado à intempérie; foi realizado um seguimento semanal dos itens mencionados mediante observação direta dos dois grupos de plantações.

Resultados: durante as primeiras cinco semanas, apresentou-se um desenvolvimento similar; a partir da sétima semana, o cultivo sob abrigo apresentou 38\% de maior quantidade de ramos, igualdade no âmbito de frutos e $28 \%$ de maior altura; na semana 13, as diferenças foram elevadas a $64 \%$ em quantidades de ramos, $65 \%$ de maior quantidade de frutos e 55\% em altura.

Conclusão: comprovou-se que a implantação dessa solução tecnológica pode propiciar um aumento do índice de crescimento e produção atingido pelas plantas.

Originalidade: desenvolvimento de um sistema eletrônico para o setor agricultor de uma região intermediária da Colômbia, com o propósito de melhorar a eficiência dos plantios.

Limitações: a carência de acesso à internet impossibilitou a implantação de um sistema controle via web.

Palavras-chave: atuadores, automatização, cultivos, estufa, sensores, software. 


\section{Introducción}

\subsection{Antecedentes}

La comunidad académica ha realizado trabajos encaminados a la medición de factores climáticos en los invernaderos. Prueba de esto es el denominado "Perfiles de temperatura y humedad relativa dentro del invernadero de la unidad agroecológica La Aldana de la Universidad del Quindío", cuyo objetivo fue el análisis preliminar de las condiciones microclimáticas del invernadero ubicado en la unidad agroecológica La Aldana de la misma institución. Tal como se menciona en [1], el invernadero consta de un sistema de instrumentación electrónica basado en sensores, el cual envía la información a un programa en Labview con el propósito de almacenar la información y, posteriormente, analizarla a fin de establecer el comportamiento de la temperatura y la humedad relativa dentro del invernadero.

La Universidad Tecnológica del Suroeste de Guanajuato es otro ejemplo de aplicación de estos proyectos, ya que ha desarrollado aplicaciones para sus invernaderos, los cuales no contaban con sistemas de control ni automatización del microclima y, por lo tanto, no les era posible regular las condiciones ambientales necesarias de acuerdo con el tipo de cultivo establecido. Rodríguez, Chagolla y M. López [2] afirman que los sistemas agrícolas automatizados están equipados para realizar un control de la temperatura del aire y de las raíces, de la intensidad luminosa, del agua y las múltiples variables del ambiente; este control protege a los sistemas agrícolas de los climas adversos y crea así un ambiente artificial o microclima, el cual ofrece las mejores condiciones de crecimiento y de desarrollo para las plantas.

Asimismo, profesores de la Universidad de Ciencia y Tecnología de China desarrollaron un trabajo de diseño e implementación de una red de monitoreo y control inalámbrico por medio de la tecnología Zigbee. De esta forma trataron de resolver los problemas de precisión, expansibilidad y costos del sistema tradicional de cableado. Como se menciona en [3], los nodos del sensor inalámbrico ZigBee recopilan datos en tiempo real en el invernadero, para luego enviarlos a un equipo que muestrea los datos.

En este mismo sentido, algunos docentes de la Universidad West Anhui (China) realizaron un artículo acerca de un sistema de iluminación PWM basado en lazo cerrado para un invernadero, con el propósito de solucionar los problemas de iluminación de los sembradíos. C. Lu, G. Zhang, C. Du y J. Cheng [4] afirman que el valor de iluminación se establece de acuerdo con diversos niveles necesarios para el cultivo en diferentes periodos. La unidad de control central realizará un análisis contrastivo a fin de amplificar o reducir el pulso de controlador PWM.

\subsection{Preliminares}

El clima a nivel mundial ha cambiado de forma drástica. Prueba de esto es el surgimiento de una serie de problemáticas de índole ambiental tales como heladas, grandes tormentas, incremento de radiación solar, sequías extremas, etc., que han alterado el estilo de vida de la humanidad, especialmente la forma en la cual se desarrollan los procesos en la agricultura. Como se mencionó en [5], el cambio climático ha planteado una cantidad de desafíos para la agricultura de diversos países; esto debido a las variaciones de producción y costos asociados con este fenómeno.

Esta afectación de la producción agrícola genera a su vez un detrimento de las ganancias y la competitividad de los agricultores a nivel local y global; además, en [6] se afirma que estas pérdidas de productividad rural están directamente relacionadas con el clima alrededor del mundo, y pueden cambiar o revertir las ventajas comparativas entre las regiones.

Como solución a esta problemática, surgieron una serie de alternativas de solución, una de las cuales se fundamenta en la implementación de una protección física de los cultivos por medio de invernaderos; en [7] se indica que esta tecnología ha permitido resguardar los sembradíos de las condiciones climáticas adversas, permitiendo producción durante todo el año, así como una gestión de protección para un mejor control de plagas y enfermedades.

Por lo general, el control de las condiciones climáticas al interior de invernaderos se realiza de forma manual, situación que puede acarrear una serie de escenarios perjudiciales para el cultivo: excesivo suministro de agua por lectura inadecuada de humedad del suelo, incremento de temperatura que afecta la transpiración de las plantas, dificultad en la toma de nutrientes, etc. 
De esta forma, se hace necesario establecer un control automático de variables ambientales al interior de un invernadero por medio de sistemas de monitoreo y control que incluyan: tarjetas de desarrollo, software, sensores y actuadores. En este sentido, [8] menciona que la acción de automatizar un invernadero consiste en implementar un sistema adecuado que sea capaz de optimizar la calidad e incrementar la eficiencia de los cultivos.

Por medio del presente trabajo, se buscó comprobar si la implementación de un sistema de automatización y control de variables climáticas al interior de un invernadero podría mejorar la eficiencia de los cultivos. Lo anterior en busca de dar solución a las problemáticas económicas y ambientales que trae consigo la posible inexactitud en las cantidades de agua, calor y luminosidad suministradas al cultivo al interior de un invernadero.

\section{Metodología}

\subsection{Participantes}

Parte de la información necesaria para el desarrollo del presente trabajo fue suministrada por agricultores que poseían cultivos bajo cubierta en el municipio de Gigante (Huila). De esta forma, al emplear muestreo no probabilístico y criterios de exclusión (seguridad para desplazamiento, lugares alejados y de difícil acceso) se estableció un tamaño de muestra de 10 personas, a quienes se les realizó una entrevista.

\subsection{Datos obtenidos}

Material de construcción invernadero: $75 \%$ guadua, y $25 \%$ estructuras metálicas; en la figura 1, se observan los resultados.

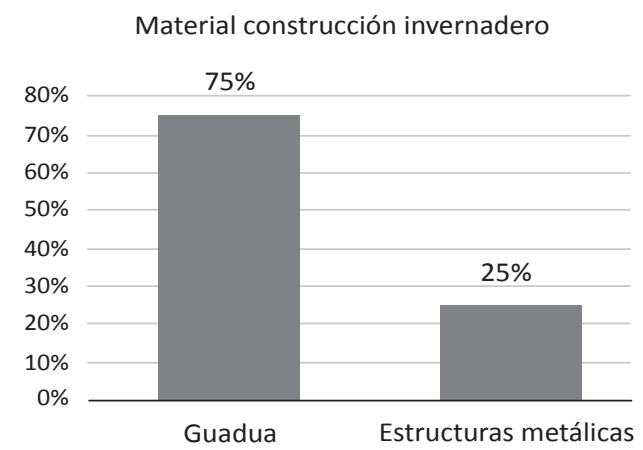

Figura 1. Material construcción invernadero Fuente: elaboración propia
Control de riego: $50 \%$ sin control, $25 \%$ control por cantidad de litros, y $25 \%$ control por tiempo de riego; en la figura 2, se observan los resultados.

\section{Control Riesgo Cultivo}

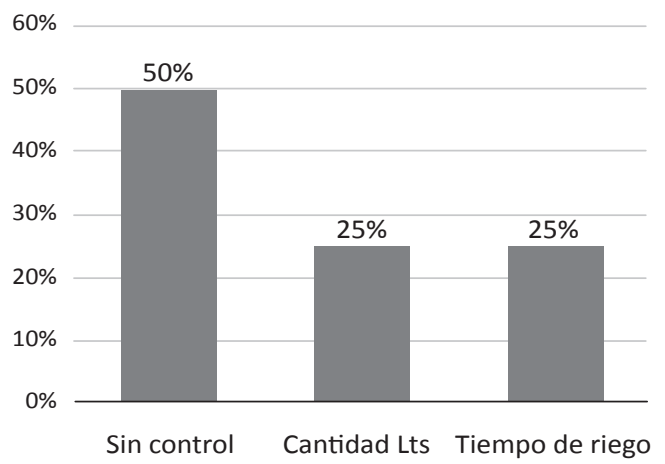

Figura 2. Riego en el cultivo

Fuente: elaboración propia

Control de humedad: 100\% no realiza, y 0\% realiza; en la figura, 3 se observan los resultados.

Control de humedad

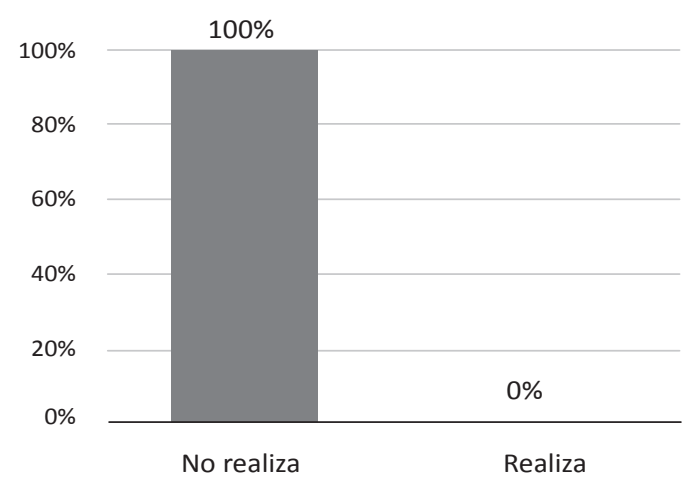

Figura 3. Control humedad

Fuente: elaboración propia

Control de temperatura: $100 \%$ no realiza, $0 \%$ realiza; en la figura 4 , se observan los resultados. 
Control de temperatura

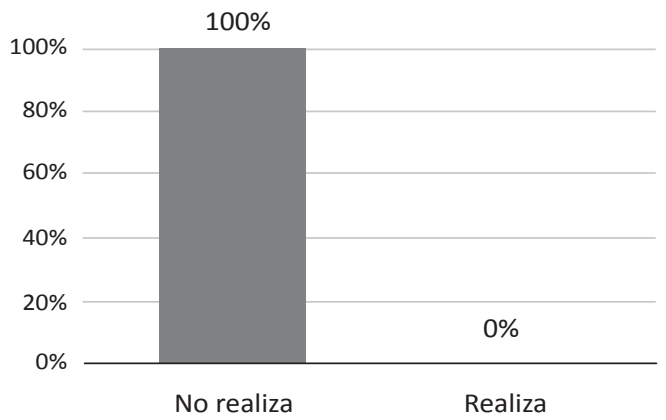

Figura 4. Control temperatura

Fuente: elaboración propia

Invernadero con procesos automatizados: $100 \%$ no tiene, y $0 \%$ tiene; en la figura 5 , se observan los resultados.

Automatización invernadero

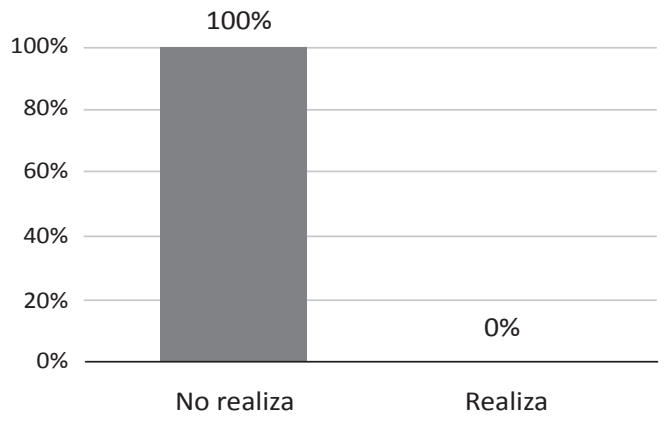

Figura 5. Automatización de invernadero Fuente: elaboración propia

\subsection{Desarrollo}

\subsubsection{Construcción de prototipo de invernadero}

A fin de realizar pruebas al sistema de automatización y control de variables ambientales, se construyó un invernadero tipo capilla a escala; este se instaló en las locaciones de la sede rural de la Corporación Universitaria del Huila, ubicada en el municipio de Palermo (Huila). El montaje consistió en una estructura de las siguientes dimensiones: 3 $\mathrm{m}$ de ancho, $3 \mathrm{~m}$ de largo y 2,5 $\mathrm{m}$ de alto. En la figura 6 , se observa una imagen del prototipo de invernadero ya terminado.

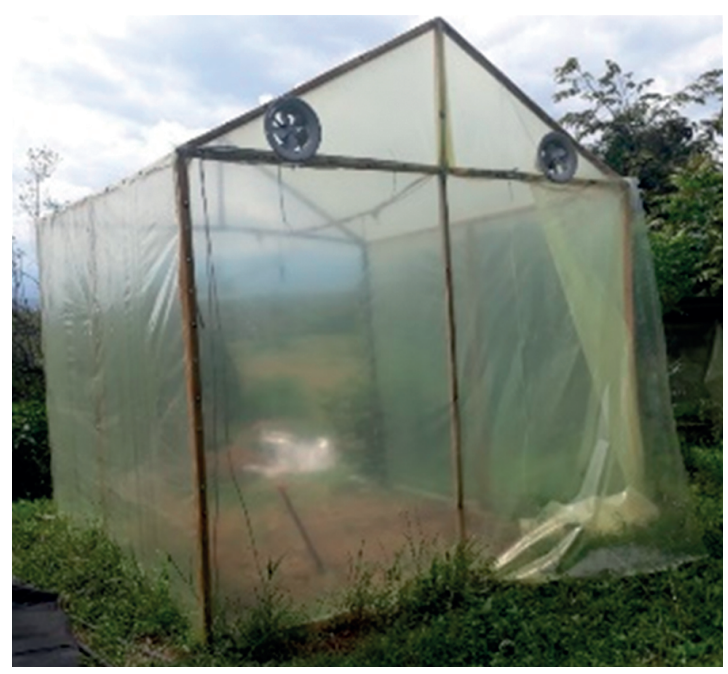

Figura 6. Prototipo invernadero Fuente: elaboración propia

\subsubsection{Nodo sensor, nodo coordinador $y$ nodo de potencia}

En concordancia con lo que se señala en [9], se puede afirmar que el nodo sensor es el responsable de recolectar las señales externas provenientes de los sensores y enviarlas hacia el nodo coordinador gracias a un transmisor RF.

A su vez, el nodo coordinador es un dispositivo concentrador y de enrutamiento de señales provistas por nodos sensores ubicados alrededor de los cultivos. En [10] se mencionó anteriormente que este está encargado de establecer el canal de comunicaciones y encaminar paquetes; además es el encargado de procesar los datos y enviar ordenes al nodo de potencia.

Este último es el encargado de recibir la señal transmitida desde el nodo coordinador, con el propósito de realizar el proceso ON-OFF de los actuadores dispuestos en el invernadero.

Como central de procesamiento de datos de nodo sensor, nodo coordinador y nodo de potencia, se seleccionó la placa Arduino Leonardo; a su vez, para la comunicación entre estos se eligió la tecnología XBEE, la cual trabaja con base en el protocolo 
de comunicaciones para redes PAN ZigBee (IEEE 802.15.4). Este, según [11], es el mejor estándar para el monitoreo de la agricultura debido al bajo consumo de potencia para aplicaciones a gran escala; en la figura 7 , se observa parte de la conexión del nodo de potencia: etapa de control, etapa de potencia, etapa de transmisión.

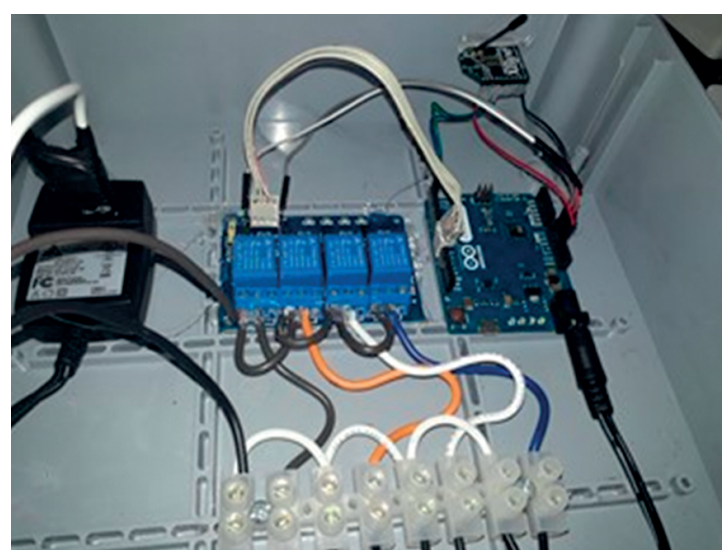

Figura 7. Nodo de potencia

Fuente: elaboración propia

La selección de sensores y actuadores del sistema de automatización se realizó de la siguiente forma:

- Sensores: Sht71 (temperatura y humedad relativa), LDR (fotocelda), y HL-69 (humedad de suelo). En [5] se afirma que estos dispositivos son los encargados de recolectar los valores de las variables ambientales y del suelo de la zona de cultivo, así como de transmitirlas hasta el nodo sensor.

- Actuadores: unidad extractora de aire, unidad inyectora de aire, nebulizadores, lámparas alógenas y motobomba (control de humedad relativa y sistema de riego). Según [10], estos dispositivos son un conjunto de elementos de accionamiento necesarios para modificar las condiciones ambientales del invernadero.

\subsubsection{Estación base}

La estación base es un computador de mesa que tiene instalado el software de control proporcional y monitoreo para invernadero. Por medio de este, se realiza un análisis de los datos provistos por el nodo coordinador y se ejecutan actividades de procesamiento para posteriormente enviar señales de control a través del nodo anteriormente mencionado. El código fuente del software de control se genera en Visual Basic 2008, y los datos se transmiten al módulo Xbee del nodo coordinador de forma serial para posteriormente establecer conexión con el nodo sensor y el nodo de potencia con el propósito de intercambiar órdenes.

\subsubsection{Topología}

Además, se seleccionó la topología de conexión estrella debido a su facilidad de configuración y sus bajos costos de implementación. Vela [8] establece que la topología tipo malla ofrece mayor confiabilidad debido a los múltiples caminos que existen para comunicar los nodos; en la figura 8 se observa una imagen de la red utilizada.

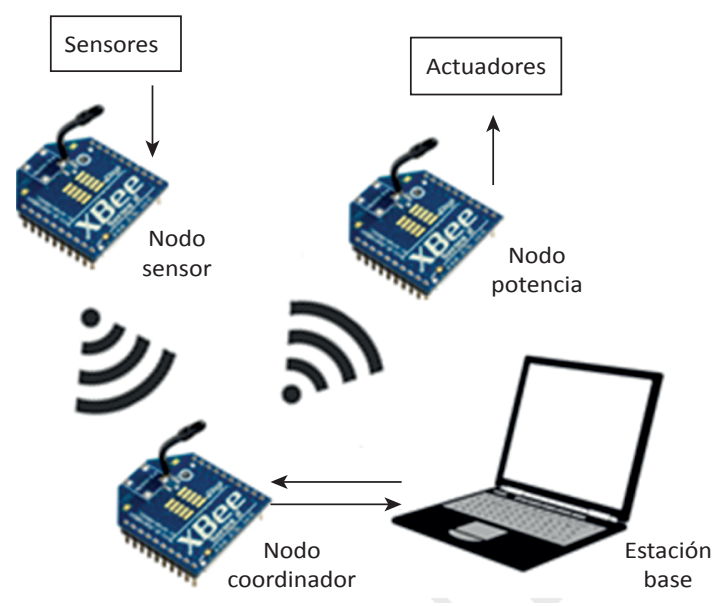

Figura 8. Topología

Fuente: elaboración propia

\subsubsection{Cultivo}

Debido a su adaptación a una gran variedad de suelos, se seleccionó el tomate como la planta a cultivar al interior del invernadero. Además, el DANE [12] afirma que este presenta un mejor comportamiento en suelos sueltos, aireados, bien drenados y con buena capacidad de retención de humedad.

Las condiciones agroecológicas ideales para el cultivo del tomate de mesa según lo mencionó anteriormente el DANE [12] son: temperatura de 16 a 20 ${ }^{\circ} \mathrm{C}$ para el periodo nocturno, y de 22 a $30{ }^{\circ} \mathrm{C}$ en el 
diurno, humedad relativa de 50 a $65 \%$, y de ocho a 16 horas diarias de luz solar.

Se realizaron dos tipos de plantaciones: una al interior del invernadero denominada "cultivo experimental", y otra en las afueras del invernadero denominada "cultivo de control". Esto con el propósito de realizar una medición y comparación constante del crecimiento, así como una validación de la eficiencia del sistema de control.

\section{Resultados}

Inicialmente se realizó una prueba de toma de datos de los sensores en un terrero sin plantación con el objetivo de verificar la correcta lectura de la información. Esta se recopiló y almacenó cada 10 minutos en una base de datos en Access, con el propósito de realizar un seguimiento al comportamiento de las variables ambientales una vez entrara en funcionamiento el sistema de automatización y control. En la tabla 1, se observa que existe una medición continua de las condiciones climáticas del invernadero.
Posteriormente, se desarrolló una prueba de 24 horas con el objetivo de evaluar la durabilidad de la batería en los nodos sensor y el nodo de potencia, así como la estabilidad del software. En la figura 9, se observa la continuidad de medición y control de temperatura a lo largo del día.

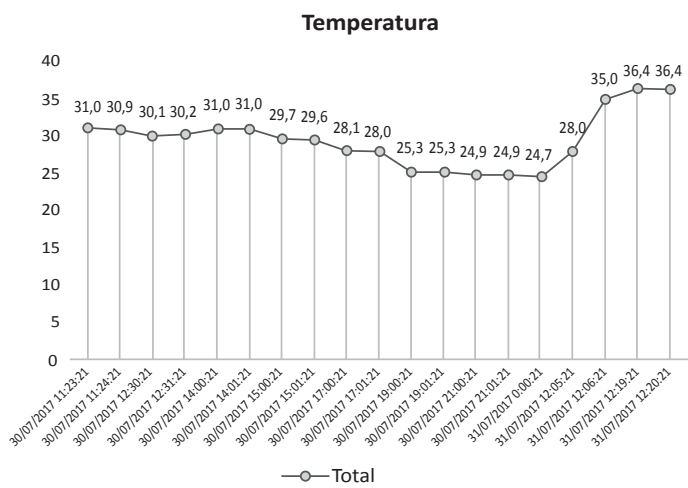

Figura 9. Resultados temperatura

Fuente: elaboración propia

En la figura 10, se observa la continuidad de medición y control de humedad relativa del cultivo a lo largo del día.

Tabla 1. Resultado de recolección de datos

\begin{tabular}{|c|c|c|c|c|c|c|c|c|}
\hline Hora & Temperatura & Humedad & Iluminosidad & HSuelo & Extractores & Riego & Iluminación & Polisombra \\
\hline $\begin{array}{c}30 / 07 / 2017 \\
11: 23: 21\end{array}$ & 31 & $48,1 \%$ & 3725 & $81,8 \%$ & ON & OFF & OFF & OFF \\
\hline $\begin{array}{c}30 / 07 / 2017 \\
12: 30: 21\end{array}$ & 30,1 & $54,2 \%$ & 5622 & $76,6 \%$ & ON & OFF & OFF & OFF \\
\hline $\begin{array}{c}30 / 07 / 2017 \\
14: 01: 21\end{array}$ & 31 & $52,8 \%$ & 4809 & $37,9 \%$ & ON & ON & OFF & OFF \\
\hline $\begin{array}{c}30 / 07 / 2017 \\
15: 00: 21\end{array}$ & 29,7 & $54,8 \%$ & 3346 & $31,1 \%$ & OFF & ON & OFF & OFF \\
\hline $\begin{array}{c}30 / 07 / 2017 \\
17: 01: 21\end{array}$ & 28 & $57,9 \%$ & 2777 & $23,1 \%$ & OFF & ON & OFF & OFF \\
\hline $\begin{array}{c}30 / 07 / 2017 \\
19: 01: 21\end{array}$ & 25,3 & $61,4 \%$ & 0 & $19,5 \%$ & OFF & ON & OFF & OFF \\
\hline $\begin{array}{c}30 / 07 / 2017 \\
21: 01: 21\end{array}$ & 24,9 & $62,1 \%$ & 0 & $16,2 \%$ & OFF & ON & OFF & OFF \\
\hline $\begin{array}{c}31 / 07 / 2017 \\
0: 00: 21\end{array}$ & 24,7 & $63,1 \%$ & 0 & $15,7 \%$ & OFF & ON & OFF & OFF \\
\hline $\begin{array}{c}31 / 07 / 2017 \\
12: 05: 21\end{array}$ & 28 & $21,3 \%$ & 8466 & $55 \%$ & ON & OFF & OFF & OFF \\
\hline $\begin{array}{c}31 / 07 / 2017 \\
12: 20: 21\end{array}$ & 36,4 & $56,5 \%$ & 9685 & $44,2 \%$ & ON & ON & OFF & OFF \\
\hline
\end{tabular}

Fuente: elaboración propia 


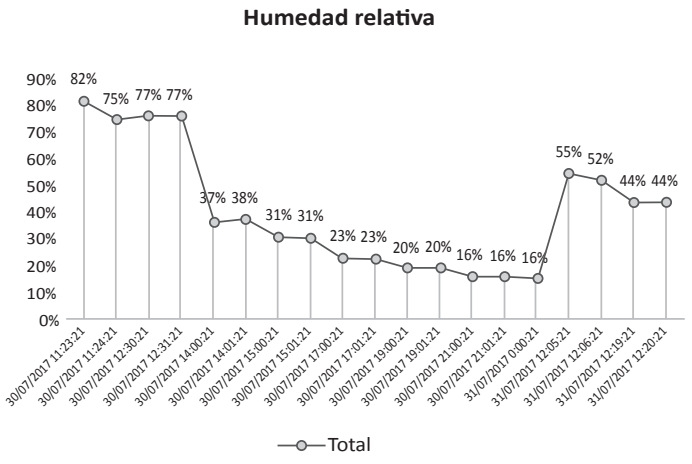

Figura 10. Resultados humedad relativa

Fuente: elaboración propia

En la figura 11, se observa la continuidad de medición y control de luminosidad del cultivo a lo largo del día.

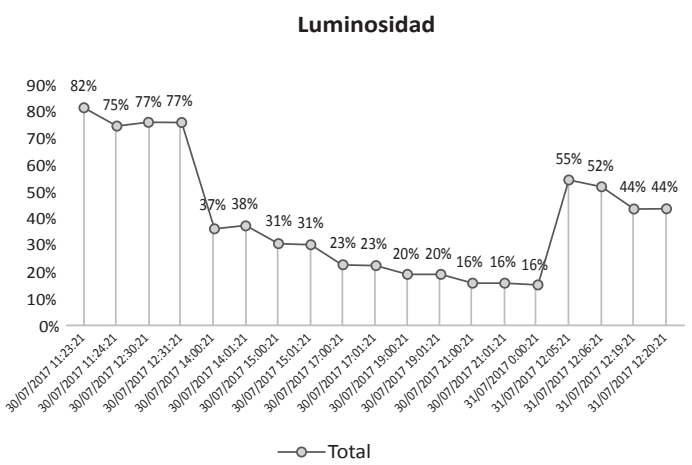

Figura 11. Resultados luminosidad

Fuente: elaboración propia

En la figura 12, se observa la continuidad de medición y control de humedad del suelo a lo largo del día.

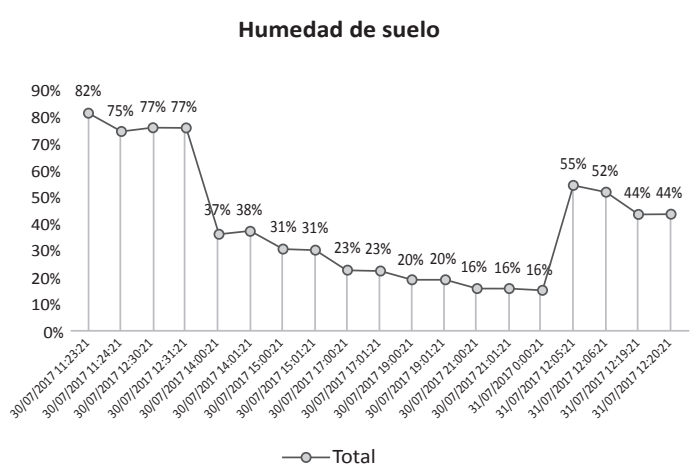

Figura 12. Resultados humedad del suelo Fuente: elaboración propia
Una vez realizada la siembra de las plantaciones de tomate, se desarrolló un proceso de recolección de información por un periodo de nueve semanas; durante este lapso, se tomaron mediciones con frecuencia semanal. Cabe destacar que tanto el cultivo experimental como el de control se plantaron en las mismas condiciones y se les suministraron los mismos cuidados iniciales.

En este periodo, se efectuaron mediciones de número de ramas, altura de las plantas y aspecto general de los frutos, tanto para el cultivo bajo cubierta (experimental) como para el ubicado en el exterior del invernadero (de control). A continuación, se presentan los resultados obtenidos y las gráficas comparativas entre las dos plantaciones.

\subsection{Número de ramas}

Durante las primeras cinco semanas, se observó un comportamiento similar en las plantaciones bajo cubierta y exterior, lo cual pudo ser producto de las condiciones naturales de crecimiento del tomate. A partir de la séptima semana, se presentó una diferencia notable en el crecimiento del cultivo ubicado al interior del invernadero; esta tendencia continuó, y en cada medición se acentuó aún más el desfase de desarrollo entre los sembradíos estudiados.

De esta forma, en la semana nueve se logró constatar una gran discrepancia en el número de ramas: 21 para el cultivo bajo cubierta y 13 para el exterior. Esto permitió comprobar que el plantío intervenido presentaba un mayor progreso; en la figura 13 , se visualiza la situación anteriormente expuesta.

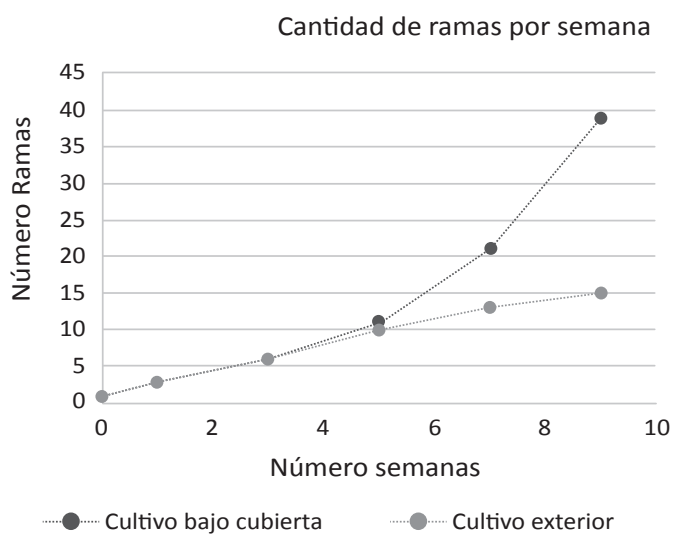

Figura 13. Número de ramas de las dos plantaciones Fuente: elaboración propia 


\subsection{Altura de la planta}

Durante las cinco primeras semanas, se presentaron unas pequeñas discrepancias de altura de planta entre el cultivo bajo cubierta y el que estaba ubicado en el exterior. Una vez cumplida la semana siete, se pudo constatar que el cultivo bajo cubierta presentó un crecimiento superior al cultivo ubicado en el exterior, ya que las plantas del primero poseían una altura de $67 \mathrm{~cm}$ y las del segundo unos $48 \mathrm{~cm}$, en promedio, es decir, una diferencia de cerca de $19 \mathrm{~cm}$; posteriormente, en la semana nueve, la diferencia se incrementó a $36 \mathrm{~cm}$; en la figura 14 se visualiza esta situación.

Altura de planta

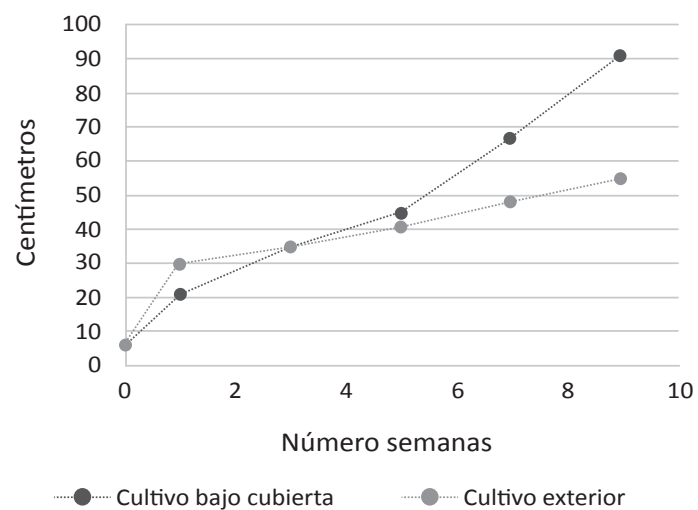

Figura 14. Altura de las plantas

Fuente: elaboración propia

\subsection{Fruto}

Durante las tres primeras semanas, no se observaron frutos en ninguna de las dos plantaciones; sin embargo, en la semana cinco, se presentó un fruto en promedio en las plantas que se encontraba en el exterior. Posteriormente, hacia la novena semana, las plantas de los sembradíos contaban con tres frutos; sobre la semana 11, el cultivo ubicado al interior del invernadero exhibió 10 frutos y el exterior, solo cuatro; finalmente, la diferencia creció hasta 17 frutos bajo cubierta y solo seis en el exterior. En la figura 15 , se visualiza esta situación.

En la tabla 2, se observan los datos obtenidos del cultivo experimental y de control.

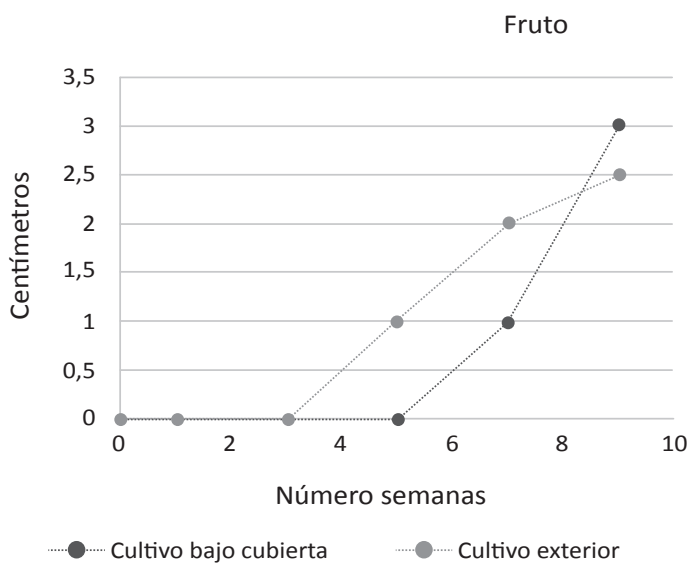

Figura 15. Frutos

Fuente: elaboración propia

Tabla 2. Resultados del crecimiento del cultivo

\begin{tabular}{|c|c|c|c|c|c|c|c|}
\hline \multicolumn{8}{|c|}{ Cultivo bajo cubierta (experimental) } \\
\hline Semana & 1 & 3 & 5 & 7 & 9 & 11 & 13 \\
\hline Altura $(\mathrm{cm})$ & 21 & 35 & 45 & 67 & 91 & 140 & 180 \\
\hline Ramas (unidad) & 3 & 6 & 10 & 21 & 39 & 54 & 89 \\
\hline Frutos por planta (Promedio) & 0 & 0 & 0 & 1 & 3 & 10 & 17 \\
\hline \multicolumn{8}{|c|}{ Cultivo exterior (de control) } \\
\hline Semana & 1 & 3 & 5 & 7 & 9 & 11 & 13 \\
\hline Altura (cm) & 30 & 35 & 41 & 48 & 55 & 62 & 82 \\
\hline Ramas (unidad) & 3 & 6 & 10 & 13 & 15 & 27 & 32 \\
\hline Frutos por planta (promedio) & 0 & 0 & 1 & 1 & 3 & 4 & 6 \\
\hline
\end{tabular}

Fuente: elaboración propia 


\section{Discusión}

En primer lugar, se puede afirmar que la información provista por los agricultores de la región central del Huila que poseían cultivos bajo cubierta permitió constatar que estos no utilizaban ningún tipo de dispositivo electrónico para el control de variables ambientales al interior del invernadero; por tanto, se presentaba una marcada incertidumbre acerca de los valores de ET en el interior del invernadero, como consecuencia derivada de la intervención manual de estas.

Asimismo, se pudo comprobar que se presentó un mayor desarrollo tanto a nivel de tamaño (altura y cantidad de ramas) como de cantidad de frutos promedio de las plantas ubicadas al interior del invernadero con respecto a aquellas situadas en su exterior. Esto se pudo constatar gracias a las mediciones semanales que se realizaron sobre el cultivo experimental y el de control.

En este mismo sentido, se logró comprobar que la implementación del sistema de control automático de variables climáticas permitió efectuar un control estricto de la cantidad de agua suministrada al cultivo, de manera que se logró reducir los problemas de deshidratación de la planta por falta de líquido o de asfixia radicular por exceso de este; asimismo, gracias a la automatización de funcionamiento de extractores de aire fue posible establecer un nivel adecuado de temperatura al interior del invernadero y crear así un ambiente ideal para que el cultivo experimental se desarrollara con normalidad y en menos tiempo que el de control.

Además, gracias a las pruebas y mediciones realizadas en el cultivo experimental y el de control, se pudo comprobar que, debido a la intervención del sistema de control automático de las variables ambientales que influyen en el desarrollo de los cultivos, se logró un mayor crecimiento de las plantas y en menor tiempo, lo cual podría acarrear una posible reducción en los costos asociados a compra de abonos y pesticidas.

De igual forma, este trabajo permitió comprobar que el adelanto de actividades de investigación ligado al desarrollo de las actividades económicas diarias de una región en particular podría a futuro influir en el crecimiento productivo y social del sector agrícola de un determinado territorio, en este caso y de forma especial en el departamento del Huila.
Por otra parte, una de las principales limitaciones que se presentaron al momento de desarrollar el presente trabajo fue la carencia de acceso a Internet en el sitio de construcción del invernadero, situación que imposibilitó la implementación de un sistema web de control de variables ambientales; esto debido a que la ubicación de la finca a las afueras de la ciudad dificultó la instalación de un servicio de red que garantizara una conexión constante al servidor. Por tanto, como consecuencia de lo anterior se optó por desarrollar un software de escritorio que permitiera ejecutar las actividades de automatización a nivel local.

\section{Conclusiones}

En la actualidad, los cambios a nivel climático han propiciado que los agricultores generen estrategias con el propósito de reducir posibles afectaciones de los cultivos. De esta forma, los invernaderos surgen como una alternativa que permite aislar las plantaciones y, al mismo tiempo, generar un ambiente ideal para el desarrollo de estas. Los cultivadores intentan realizar control de las variables ambientales al interior de los cultivos, lo cual en la mayoría de los casos se efectúa de forma manual y experimental; esta situación puede generar una necesidad mayor de recursos hídricos y energéticos.

En este mismo sentido, el presente artículo muestra cómo la implementación de tecnología de automatización y control al interior de un invernadero podría solucionar problemáticas económicas y ambientales de la población anteriormente descrita, ya que el sistema asegura unas condiciones ambientales ideales para el cultivo bajo cubierta sin la intervención directa del agricultor.

Esta situación se pudo comprobar mediante la realización de las pruebas descritas en este documento, en las cuales se dispuso de un sistema automático para el control de variables ambientales al interior de un prototipo de invernadero tipo capilla, ubicado en las locaciones de la finca de la Corporación Universitaria del Huila en el municipio de Palermo; este diseño se acogió debido a la baja complejidad de la arquitectura, los bajos costos asociados con la edificación y la facilidad de acceso a materiales a nivel local.

A su vez, la arquitectura del sistema de control anteriormente descrito se basó en una topología tipo estrella: nodo sensor, nodo potencia y nodo 
de control, los cuales establecían constante comunicación con el propósito de realizar los procesos automáticos de control de variables ambientales al interior del invernadero. Los nodos se programaron de forma independiente a fin de realizar procesos más agiles de actualización de software de cada uno de ellos.

Además, se pudo comprobar que los módulos XBEE proporcionaron una comunicación eficiente entre los nodos del sistema de control, lo cual permitió que el envío y la recepción de mensajes se realizara en tiempo real, logrando de esta forma un nivel ideal de las variables ambientales al interior del invernadero. Asimismo, la interfaz de usuario diseñada permitió que el usuario lograra seleccionar las condiciones ideales para el cultivo específico (en este caso el tomate), así como realizar un seguimiento constante de sus cambios climáticos.

\section{Referencias}

[1] P. Muñoz y J. Buitrago, "Perfiles de temperatura y humedad relativa dentro del invernadero de la unidad agroecológica La Aldana de la Universidad del Quindío", IngEam, vol. 2, n. ${ }^{\circ}$ 2, pp. 90-114, 2015. [En línea]. Disponible en: http://www.eam.edu.co/ojs/ index.php/ingeam/article/view/96/103

[2] M. Rodríguez, H. Chagolla y M. López, "Diseño conceptual de sistema para la automatización del invernadero uno de la Universidad Tecnológica del Suroeste de Guanajuato", Ciencias de la Ingeniería y Tecnología Handbook T-IV: Congreso Interdisciplinario de Cuerpos Académicos, pp. 299-318, 2014. [En línea]. Disponible en: http://ecorfan.org/handbooks/Ciencias\%20de\%20la\%20Ingenieria\%20 y\%20Tecnologia\%20TV/Ciencias\%20de\%20la\%20 Ingenier\%C3\%ADa\%20y\%20Tecnolog\%C3\%ADa \%20Handbook\%20T_V.pdf

[3] F. Chen, L. Qin, X. Li, G. Wu y C. Shi, "Design and implementation of ZigBee wireless sensor and control network system in greenhouse", 2017 36th Chinese Control Conference (CCC), Dalian, 2017, pp. 8982-8986. doi: 10.23919/ChiCC.2017.8028786

[4] C. Lu, G. Zhang, C. Du y J. Cheng, "Design of closed-loop feedback control system for mini greenhouse illumination based on PWM", 2017 32nd Youth Academic Annual Conference of Chinese Association of Automation (YAC), Hefei, 2017, pp. 541-543. doi: 10.1109/YAC.2017.7967469
[5] C. L. Walthall et al., "Climate change and agriculture in the United States: effects and adaptation", USDA Technical Bulletin, no. 1935, Feb. 2013, p. i-186, 2013. [En línea]. Disponible en: https://www.usda.gov/ oce/climate_change/effects_2012/CC\%20and $\% 20$ Agriculture\%20Report\%20(02-04-2013)b.pdf

[6] A. Calzadilla, T. Zhu, K. Rehdanz, R. S. J. Tol y C. Ringler, "Economywide impacts of climate change on agriculture in Sub-Saharan Africa", Ecol. Econ., vol. 93, pp. 150-165, 2013. doi: 10.1016/j.ecolecon.2013.05.006

[7] W. Baudoin, R. Nono-Womdim, N. Lutaladio, A. Hodder, N. Castilla, C. Leonardi y R. Duffy, "Good agricultural practices for greenhouse vegetable crops: principles for Mediterranean climate areas", FAO Plant production and protection paper-greenhouse design and covering materials, 2013. [En línea]. Disponible en: http://www.fao.org/docrep/018/i328 4e/i3284e.pdf\#page $=79$

[8] A. Vela, "Estudio de la agricultura de precisión enfocado en la implementación de una red de sensores inalámbricos (WSN) para el monitoreo de humedad y temperatura en cultivos-caso de estudio Hacienda Cabalinus ubicada en la provincia de Los Ríos", Revista Politécnica, vol. 38, n. ${ }^{\circ}$ 1, 2016. [En línea]. Disponible en: http://repositorio.puce.edu.ec/bitstream/handle/ 22000/11112/Art\%c3\%adculo\%20 cient\%c3\%adfico\%20Andr\%c3\%a9s\%20Vela-Revista $\% 20 E P N . P D F$ ? sequence $=1$ \&isAllowed $=y_{\text {_ }}$

[9] A. Cama, Fr. Gil, J. Gómez, A. García y F. Manzano, "Sistema inalámbrico de monitorización para cultivos en invernadero", Dyna, vol. 81, n. ${ }^{\circ} 184$, pp. 164170, 2014. doi: 10.15446/dyna.v81n184.37034

[10] N. D. Castro C., L. E. Chamorro F. y C. A. M. Viteri, "Una red de sensores inalámbricos para la automatización y control del riego localizado", Rev. Ciencias Agrícolas, vol. 33, n. ${ }^{\circ}$ 2, p. 106, 2016. doi: 10.22267/ rcia. 163302.57

[11] J. Cedeño, M. Zambrano y C. Medina, "Redes inalámbricas de sensores eficientes para la agroindustria”, Prisma Tecnológico, vol. 5, pp. 22-25, 2014. [En línea]. Disponible en: http://www.revistas.utp.ac.pa/ index.php/prisma/ article/download/518/513

[12] DANE, "El cultivo del tomate de mesa bajo invernadero, tecnología que ofrece mayor producción, calidad e inocuidad del producto", Boletín Mensual-insumos y factores asociados a la producción agropecuaria, p. 72, 2014. [En línea]. Disponible en: https://www. dane.gov.co/files/investigaciones/agropecuario/sipsa/insumos_factores_de_produccion_dic_2014.pdf 